\title{
SUR LA STRUCTURE DU GROUPE D'AUTOMORPHISMES DE CERTAINES SURFACES AFFINES
}

\author{
StÉPHANE LAMY
}

\begin{abstract}
We describe the structure of the group of algebraic automorphisms of the following surfaces 1) $\mathbb{P}_{k}^{1} \times \mathbb{P}_{k}^{1}$ minus a diagonal; 2) $\mathbb{P}_{k}^{1} \times \mathbb{P}_{k}^{1}$ minus a fiber. The motivation is to get a new proof of two theorems proven respectively by L. Makar-Limanov and H. Nagao. We also discuss the structure of the semi-group of polynomial proper maps from $\mathbb{C}^{2}$ to $\mathbb{C}^{2}$
\end{abstract}

\section{Introduction}

Dans $[6]$ nous avons montré comment retrouver via des arguments géométriques le théorème classique de Jung-Van der Kulk décrivant la structure des automorphismes du plan affine $\mathbb{A}_{k}^{2}(k$ étant un corps quelconque) :

Théorème 1 (Jung-Van der Kulk). Le groupe des automorphismes du plan affine $\mathbb{A}_{k}^{2}$ est le produit amalgamé du groupe affine

$A=\left\{(x, y) \mapsto\left(a_{1} x+b_{1} y+c_{1}, a_{2} x+b_{2} y+c_{2}\right) ; a_{i}, b_{i}, c_{i} \in k, a_{1} b_{2}-a_{2} b_{1} \neq 0\right\}$

et du groupe élémentaire

$$
E=\left\{(x, y) \mapsto(\alpha x+P(y), \beta y+\gamma) ; \alpha, \beta \in k^{*}, \gamma \in k, P \in k[X]\right\}
$$

le long de leur intersection.

Dans le présent article nous nous proposons de montrer comment la même démarche permet d'obtenir la description du groupe d'automorphismes d'autres classes de surfaces.

2000 Mathematics Subject Classification. 14E07.

Key words. Algebraic automorphisms, amalgamated products, polynomial proper maps. 
Nous nous sommes intéressés aux automorphismes de deux surfaces différentes de $\mathbb{A}_{k}^{2}$, à savoir $\mathbb{P}_{k}^{1} \times \mathbb{P}_{k}^{1}$ privé ou bien d'une diagonale ou bien d'une fibre. Notre motivation était de regrouper sous une même approche géométrique deux théorèmes dûs respectivement à L. Makar-Limanov et $\mathrm{H}$. Nagao. Le résultat de Makar-Limanov concerne la description des automorphismes d'une surface quadrique affine. Ce résultat (appliqué au corps de base $k=\mathbb{C}(t))$ nous a été utile dans [7] pour étudier certains automorphismes de $\mathbb{C}^{3}$. Le résultat de Nagao concerne lui la description du groupe $\mathrm{GL}(2, k[X])$. Ce théorème a été redémontré (et généralisé, à ce propos on pourra consulter $[\mathbf{9}])$ par Serre [11] en faisant agir $\mathrm{GL}_{2}(k[X])$ sur un arbre simplicial idoine.

Signalons que V. I. Danilov et M. H. Gizatullin [4] ont étudié plus généralement le groupe $G$ des automorphismes des surfaces affines admettant une compactification projective par ajout d'une courbe rationnelle; leurs résultats contiennent donc nos Théorèmes 4 et 5 . Leur démarche consiste à montrer que $G$ agit sur un arbre dont les sommets sont constitués par certaines compactifications de la surface, avec comme domaine fondamental une arête. Ils obtiennent ainsi via la théorie de Bass-Serre [11] la description de $G$ comme un produit amalgamé. Notre preuve, également de nature géométrique, est cependant assez différente. Nous utilisons la décomposition des applications birationnelles entre surfaces à l'aide d'éclatements pour exhiber des générateurs pour le groupe $G$, et constatons ensuite que l'absence de relations (i.e. la structure de produit amalgamé) n'est plus qu'une remarque immédiate (voir page 17).

Dans un dernier paragraphe nous nous intéressons à un problème un peu différent, à savoir la description de certains endomorphismes (et non plus automorphismes) du plan affine complexe. Précisément nous discutons la question de la structure du semi-groupe des applications polynomiales propres de $\mathbb{C}^{2}$ dans $\mathbb{C}^{2}$.

Tout au long de l'article nous utiliserons de manière répétée le résultat suivant, valable sur tout corps algébriquement clos (voir [5, Th. 5.5]).

Théorème 2 (Zariski). Toute application birationnelle entre deux surfaces s'obtient comme une suite d'éclatements puis de contractions; autrement dit si $X, Y$ sont des surfaces et

$$
g: X \rightarrow Y
$$


est une application birationnelle (qui n'est pas un isomorphisme), alors $i l$ existe une surface $M$ et des suites d'éclatements $\pi_{1}$ et $\pi_{2}$ tel que le diagramme suivant commute :

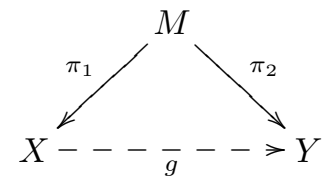

Nous nous réfèrerons au diagramme fourni par le théorème comme le diagramme de Zariski associé à $g$. Chacun des points éclatés au cours de la suite $\pi_{1}$ est appelé point d'indétermination de $g$; ces points appartiennent donc ou bien à $X$ (on dit que le point d'indétermination est propre) ou bien à une surface obtenue en éclatant $X$ (on dit alors que le point d'indétermination est dans un voisinage infiniment proche d'un point de $X^{1}$ ). Le nombre de points d'indétermination de $g$ (propres ou non) sera noté \#ind $(g)$. Nous utiliserons souvent certaines compactifications de $\mathbb{A}^{2}$ appelées surfaces de Hirzebruch, et définies par $F_{n}=\mathbb{P}\left(\mathcal{O}_{\mathbb{P}^{1}} \oplus \mathcal{O}_{\mathbb{P}^{1}}(n)\right)$. Tout ce qui nous intéresse ici est de savoir qu'une telle surface, munie d'une fibration en $\mathbb{P}^{1}$ sur $\mathbb{P}^{1}$, compactifie $\mathbb{A}^{2}$ par ajout de deux courbes rationnelles transverses : une fibre à l'infini $f_{\infty}$ et une section $s_{\infty}$ d'auto-intersection $-n$. Nous noterons $f_{\infty}\left(F_{n}\right)$ et $s_{\infty}\left(F_{n}\right)$ s'il y a ambiguité sur la surface de Hirzebruch en question. L'exemple de base est $F_{1}$ obtenue en éclatant un point $p$ sur la droite à l'infini dans $\mathbb{P}^{2}$ : dans ce cas la fibration correspond au pinceau des droites passant par $p, f_{\infty}$ est la transformée de la droite à l'infini et $s_{\infty}$ est le diviseur exceptionnel.

\section{Sur les automorphismes de $\mathbb{P}^{1} \times \mathbb{P}^{1}$ privé d'une courbe rationnelle}

Dans toute la suite $k$ sera un corps quelconque (excepté dans le cadre $\mathrm{du}$ Théorème 6 où nous supposons $\operatorname{car}(k) \neq 2)$. Nous énonçons deux théorèmes décrivant la structure des automorphismes de $\mathbb{P}^{1} \times \mathbb{P}^{1}$ privé d'une diagonale ou d'une fibre. Nous expliquons ensuite les liens avec deux théorèmes existant dans la littérature (dûs à Makar-Limanov et Nagao), avant de donner les preuves dans un dernier paragraphe.

\footnotetext{
${ }^{1} \mathrm{Si} \pi: \tilde{X} \rightarrow X$ est une suite d'éclatements, si $q$ appartient à l'un des diviseurs exceptionnels et si $\pi(q)=p \in X$, on dit que $q$ est dans un voisinage infiniment proche de $p$.
} 


\subsection{Enoncés des théorèmes.}

Notons $\left[t_{0}: t_{1}\right],\left[u_{0}: u_{1}\right]$ les coordonnées homogènes dans $\mathbb{P}^{1} \times \mathbb{P}^{1}$, et identifions $\mathbb{A}^{2}$ avec l'ouvert $t_{1} \neq 0, u_{1} \neq 0$. Nous notons $t=\frac{t_{0}}{t_{1}}, u=\frac{u_{0}}{u_{1}}$ les coordonnées dans $\mathbb{A}^{2}$. Soit $D$ la diagonale d'équation $t_{0} u_{1}+t_{1} u_{0}=0$. On appelle $A_{D}$ le groupe des automorphismes de $\mathbb{P}^{1} \times \mathbb{P}^{1} \backslash D$ qui s'étendent en des automorphismes de $\mathbb{P}^{1} \times \mathbb{P}^{1}$. Ce groupe est engendré par $(t, u) \mapsto(u, t)$ et par les automorphismes de la forme

$$
(t, u) \mapsto\left(\frac{a t+b}{c t+d}, \frac{-a u+b}{c u-d}\right) ;\left(\begin{array}{ll}
a & b \\
c & d
\end{array}\right) \in \operatorname{PGL}(2, k) .
$$

D'autre part nous désignons par $E_{D}$ le groupe des automorphismes de $\mathbb{P}^{1} \times \mathbb{P}^{1} \backslash D$ qui préservent le pinceau des droites $t+u=$ cte. $\mathrm{A}$ l'aide du Lemme 9 (voir plus loin) on peut obtenir une écriture explicite des éléments de $E_{D}$ :

Lemme 3. Le groupe $E_{D}$ est engendré par $(t, u) \mapsto(u, t)$ et par les automorphismes de la forme

$$
(t, u) \mapsto\left(\alpha t+P\left(\frac{1}{t+u}\right), \alpha u-P\left(\frac{1}{t+u}\right)\right) ; \alpha \in k^{*}, P \in k[X] .
$$

Preuve: Tout d'abord il est clair que

$A_{D} \cap E_{D}=\left\{(t, u) \mapsto(a t+b, a u-b)\right.$ ou $\left.(a u+b, a t-b) ; a \in k^{*}, b \in k\right\}$.

Maintenant soit $g \in E_{D} \backslash A_{D}$, que nous regardons comme une application birationnelle de $\mathbb{P}^{1} \times \mathbb{P}^{1}$ vers $\mathbb{P}^{1} \times \mathbb{P}^{1}$. Le pinceau engendré par les droites $t+u=$ cte contient un unique élément singulier, à savoir $\left\{t_{1}=0\right\} \cup\left\{u_{1}=0\right\}$. Ces deux droites sont donc globalement invariantes par $g$, et quitte à composer par $(t, u) \mapsto(u, t)$ on peut supposer que chacune de ces droites est invariante par $g$. De plus le point [1:0], [1:0], qui est l'unique point base du pinceau, est indéterminé ou fixe par $g$; mais cette dernière possibilité est exclue car $g^{-1}(D)$ est l'unique point d'indétermination propre de $g$ (l'unicité est donnée par le Lemme 9.1). Eclatons $[1: 0],[1: 0]$ à la source et au but, et contractons les transformées strictes de $\left\{t_{1}=0\right\}$ et $\left\{u_{1}=0\right\}$ (voir figure). 
$\mathbb{P}^{\mathbf{1}} \times \mathbb{P}^{\mathbf{1}}$
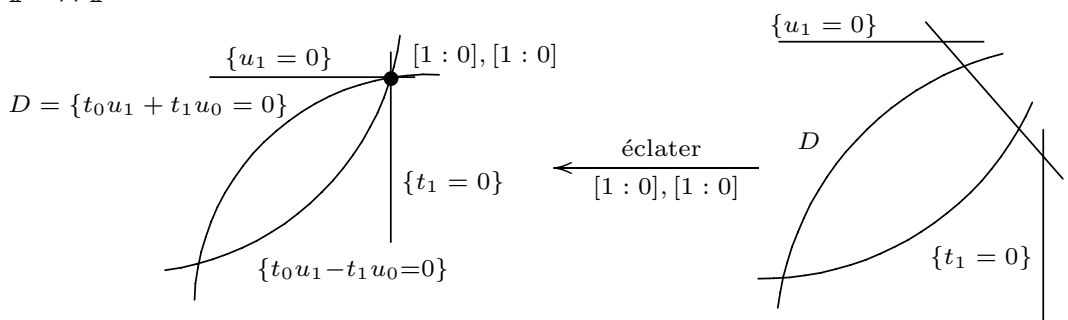

$$
\begin{gathered}
\text { contracter } \\
\left\{u_{1}=0\right\} \\
\text { et } \\
\left\{t_{1}=0\right\}
\end{gathered}
$$

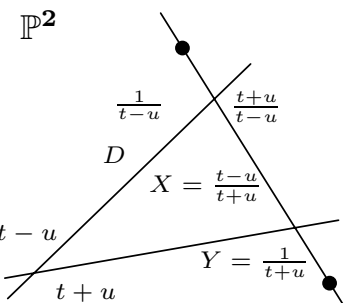

L'application $g$ est maintenant une application birationnelle de $\mathbb{P}^{2}$ dans $\mathbb{P}^{2}$, qui de plus est un automorphisme de $\mathbb{A}^{2}=\mathbb{P}^{2} \backslash D$. En effet le deuxième point d'indétermination de $g$ est encore situé sur la transformée de $D$, car celle-ci doit être l'objet de trois éclatements successifs avant de devenir d'auto-intersection -1 et de pouvoir être contractée comme l'exige l'assertion $5 \mathrm{du}$ Lemme 9. Dans la carte $X=\frac{t-u}{t+u}, Y=\frac{1}{t+u}$ on écrit donc $g$ sous la forme

$$
g:(X, Y) \mapsto(a X+P(Y), b Y+c) ; a, b \in k^{*}, c \in k, P \in k[Y] .
$$

Comme de plus la droite $\{Y=0\}$ contient deux points fixes pour $g$ (les points sur lesquels on a contracté $\left\{t_{1}=0\right\}$ et $\left.\left\{u_{1}=0\right\}\right), g$ est en fait de la forme $g:(X, Y) \mapsto(X+Y P(Y), b Y)$. En revenant aux coordonnées $t, u$ on obtient l'expression annoncée.

Théorème 4. Le groupe des automorphismes de $\mathbb{P}^{1} \times \mathbb{P}^{1} \backslash D$ est égal au produit amalgamé de $A_{D}$ et de $E_{D}$ le long de leur intersection. 
Maintenant soit $F \subset \mathbb{P}^{1} \times \mathbb{P}^{1}$ la fibre d'équation $t_{1}=0$. On note $A_{F}$ le groupe des automorphismes de $\mathbb{P}^{1} \times \mathbb{P}^{1} \backslash F$ qui s'étendent en des automorphismes de $\mathbb{P}^{1} \times \mathbb{P}^{1}$; ce groupe est constitué des automorphismes suivants :

$$
(t, u) \mapsto\left(\alpha t+\beta, \frac{a u+b}{c u+d}\right) ; \alpha \in k^{*}, \beta \in k,\left(\begin{array}{ll}
a & b \\
c & d
\end{array}\right) \in \operatorname{PGL}(2, k) .
$$

D'autre part on note $E_{F}$ le groupe des automorphismes élémentaires

$$
(t, u) \mapsto(\alpha t+\beta, \gamma u+P(t)) ; \alpha, \gamma \in k^{*}, \beta \in k, P \in k[X] .
$$

Théorème 5. Le groupe des automorphismes de $\mathbb{P}^{1} \times \mathbb{P}^{1} \backslash F$ est égal au produit amalgamé de $A_{F}$ et de $E_{F}$ le long de leur intersection.

\subsection{Un théorème de Makar-Limanov.}

Nous faisons ici le lien entre le Théorème 4 et la description du groupe $G$ des automorphismes de la surface quadrique lisse $V_{\lambda} \subset \mathbb{A}^{3}$ d'équation $y^{2}+x z=\lambda$, où $\lambda \in k^{*}$ et $\operatorname{car}(k) \neq 2$. Remarquons que dans les cas $k=0$ ou car $(k)=2$ cette surface n'est plus lisse; Makar-limanov montre que dans ce cas le groupe d'automorphismes est plus gros que dans le cas lisse : précisément il faut rajouter les homothéties de centre la singularité. Nous nous bornons ici à traiter le cas lisse.

Il existe deux sous-groupes naturels de $G$. D'une part on a le groupe orthogonal $\mathrm{O}(3, k)$ associé à la forme quadratique $y^{2}+x z$, engendré par $(x, y, z) \mapsto(x,-y, z)$ et par $\mathrm{SO}(3, k)$, ce dernier groupe étant constitué des matrices

$$
\frac{1}{a d-b c}\left(\begin{array}{ccc}
a^{2} & 2 a b & -b^{2} \\
a c & a d+b c & -b d \\
-c^{2} & -2 c d & d^{2}
\end{array}\right) \text { avec }\left(\begin{array}{ll}
a & b \\
c & d
\end{array}\right) \in \operatorname{PGL}(2, k) .
$$

Rappelons que l'identification entre $\operatorname{PGL}(2, k)$ et $\mathrm{SO}(3, k)$ peut s'obtenir en faisant agir $\operatorname{PGL}(2, k)$ par conjugaison sur les matrices $2 \times 2$ de trace nulle, que l'on identifie à $k^{3} \operatorname{par}(x, y, z) \mapsto\left(\begin{array}{cc}-y & x \\ z & y\end{array}\right)$. Cette action préserve le déterminant qui n'est rien d'autre, au signe près, que la forme quadratique $y^{2}+x z$. Remarquons que comme toute matrice dans $\mathrm{GL}(2, k)$ s'écrit comme une composition de matrices de la forme

$$
\left(\begin{array}{ll}
1 & 0 \\
c & 1
\end{array}\right),\left(\begin{array}{ll}
a & 0 \\
0 & d
\end{array}\right) \text { et }\left(\begin{array}{ll}
0 & 1 \\
1 & 0
\end{array}\right)
$$

on voit que $\mathrm{O}(3, k)$ est engendré par les matrices de la forme

$$
\left(\begin{array}{ccc}
1 & 0 & 0 \\
c & 1 & 0 \\
-c^{2} & -2 c & 1
\end{array}\right),\left(\begin{array}{ccc}
a / d & 0 & 0 \\
0 & 1 & 0 \\
0 & 0 & d / a
\end{array}\right),\left(\begin{array}{ccc}
0 & 0 & 1 \\
0 & -1 & 0 \\
1 & 0 & 0
\end{array}\right) \text { et }\left(\begin{array}{ccc}
1 & 0 & 0 \\
0 & -1 & 0 \\
0 & 0 & 1
\end{array}\right) \text {. }
$$


D'autre part on a le groupe $E_{G}$ des automorphismes de la forme ${ }^{2}$

$(x, y, z) \mapsto\left(\alpha x+2 \alpha y P(z)-\alpha z P^{2}(z), \pm(y-z P(z)), \frac{1}{\alpha} z\right) ; \alpha \in k^{*}, P \in k[X]$.

Remarquons (pour justifier l'adjectif «naturel » employé ci-dessus...) que le groupe $E_{G}$ est exactement le sous-groupe de $G$ constitué des restrictions d'automorphismes élémentaires de $\mathbb{A}^{3}$.

Théorème 6. On suppose $\operatorname{car}(k) \neq 2$. Le groupe $G$ est le produit amalgamé de $\mathrm{O}(3, k)$ et $E_{G}$ le long de leur intersection.

Ce résultat est contenu dans [8] (en fait Makar-Limanov obtient un résultat plus général concernant une classe plus large de surfaces, mais par contre ne précise pas la structure de produit amalgamé) et également dans [4]. L'énoncé ci-dessus, appliqué sur le corps $k=\mathbb{C}(t)$, était crucial dans $[7]$ où nous décrivions le groupe des automorphismes de $\mathbb{C}^{3}$ préservant la forme quadratique $y^{2}+x z$.

Nous supposons dans un premier temps que $\lambda$ admet une racine carrée $\delta$ dans $k$. Nous identifions maintenant $V_{\lambda}$ avec un ouvert de $\mathbb{P}^{1} \times \mathbb{P}^{1}$. Notons $[x: y: z: w]$ les coordonnées homogènes de $\mathbb{P}^{3}$, où l'on identifie $\mathbb{A}^{3}$ avec l'ouvert $w \neq 0$. L'équation homogénéisée de $V_{\lambda}$ devient $y^{2}-\lambda w^{2}+x z=0$. A partir du plongement de Segre

$$
\left[t_{0}: t_{1}\right],\left[u_{0}: u_{1}\right] \in \mathbb{P}^{1} \times \mathbb{P}^{1} \mapsto\left[t_{0} u_{0}: t_{1} u_{0}: t_{0} u_{1}: t_{1} u_{1}\right] \in \mathbb{P}^{3}
$$

qui identifie $\mathbb{P}^{1} \times \mathbb{P}^{1}$ avec la quadrique projective lisse $x w-y z=0$ il suffit d'un changement de variables linéaire pour obtenir la paramétrisation suivante de la quadrique $(y-\delta w)(y+\delta w)+x z=0$ (où $\left.\delta^{2}=\lambda\right)$ :

$$
\begin{aligned}
{\left[t_{0}: t_{1}\right],\left[u_{0}: u_{1}\right] } & \in \mathbb{P}^{1} \times \mathbb{P}^{1} \\
& \mapsto\left[4 \delta^{2} t_{0} u_{0}: \delta\left(t_{0} u_{1}-t_{1} u_{0}\right): t_{1} u_{1}: t_{0} u_{1}+t_{1} u_{0}\right] \in \mathbb{P}^{3} .
\end{aligned}
$$

Plus simplement, dans les cartes $(t, u)$ et $(x, y, z)$ cette paramétrisation s'écrit :

$$
(t, u) \rightarrow\left(4 \delta^{2} \frac{t u}{t+u}, \delta \frac{t-u}{t+u}, \frac{1}{t+u}\right)
$$

Via cette paramétrisation la quadrique $V_{\lambda}$ est identifiée à l'ouvert de $\mathbb{P}^{1} \times \mathbb{P}^{1}$ constitué du complémentaire de la diagonale $D=\left\{t_{0} u_{1}+t_{1} u_{0}=0\right\}$, et la fibration $z=$ cte correspond à la fibration $t+u=$ cte. De plus un

\footnotetext{
${ }^{2}$ Signalons qu'il manque un signe dans la définition de $E_{G}$ donnée dans [7], omission qui est ici réparée.
} 
calcul immédiat permet de vérifier qu'à travers cette paramétrisation l'automorphisme

$$
(t, u) \mapsto\left(t+P\left(\frac{1}{t+u}\right), u-P\left(\frac{1}{t+u}\right)\right) \in E_{D}
$$

s'identifie à

$$
(x, y, z) \mapsto\left(x-2 y(2 \delta P(z))-z(2 \delta P(z))^{2}, y+z(2 \delta P(z)), z\right) \in E_{G} .
$$

De même $(u, t) \mapsto\left(\frac{t}{c t+1}, \frac{-u}{c u-1}\right) \in A_{D}$ est identifié à $\left(\begin{array}{ccc}1 & 0 & 0 \\ -\frac{c}{2 \delta} & 1 & 0 \\ -\frac{c^{2}}{4 \delta^{2}} & \frac{c}{\delta} & 1\end{array}\right) \in$ $\mathrm{O}(3, k) ;(t, u) \mapsto(a t, a u)$ à $(x, y, z) \mapsto(a x, y, z / a),(t, u) \mapsto\left(\frac{1}{4 \delta^{2} t}, \frac{1}{4 \delta^{2} u}\right)$ à $(x, y, z) \mapsto(z,-y, x)$ et $(t, u) \mapsto(u, t)$ à $(x, y, z) \mapsto(x,-y, z)$. En conclusion le groupe $\mathrm{O}(3, k)$ s'identifie à $A_{D}$ et le groupe $E_{G}$ à $E_{D}$; ainsi le Théorème 6 est équivalent au Théorème 4 .

Supposons maintenant que $\lambda$ n'admet pas de racine carrée dans $k$; notons $\bar{k}$ la cloture algébrique de $k$ et $\delta \in \bar{k}$ une racine carrée de $\lambda$. Soit $g$ un automorphisme de $V_{\lambda}$ définie sur le corps $k$, i.e. $g$ est donné par un automorphisme de l'algèbre $k[X, Y, Z] /\left(Y^{2}+X Z-\lambda\right)$ lequel s'étend en un automorphisme de l'algèbre $k(\delta)[X, Y, Z] /\left(Y^{2}+X Z-\lambda\right)$. On peut ainsi considérer $g$ comme un automorphisme de $V_{\lambda}$ définie sur le corps $k(\delta)$. L'argument ci-dessus assure que $g$ s'écrit comme une composition d'éléments de $\mathrm{O}(3, k(\delta))$ et d'éléments de $E_{G}$ à coefficients dans $k(\delta)$. Reste à voir que ces automorphismes sont en fait à coefficients dans $k$; pour cela nous rappelons rapidement l'argument déjà développé dans [6, p. 313].

Le point crucial est que nous savons que $g$ admet un unique point d'indétermination propre, qui est l'image par $g^{-1}$ de la conique plane à l'infini (d'équations $y^{2}+x z=0$ et $w=0$ ). Choisissons un point $p$ sur $k$ de cette conique qui ne soit pas le point d'indétermination de $g^{-1}$ (l'un des deux points $[1: 0: 0: 0]$ ou $[0: 0: 1: 0]$ convient). Alors $g^{-1}(p)$ est le point d'indétermination propre de $g$, et est donc contenu dans $\mathbb{P}_{k}^{3}$. Par un raisonnement symétrique le point d'indétermination propre de $g^{-1}$ est également dans $\mathbb{P}_{k}^{3}$. En composant $g$ à droite et à gauche par des éléments bien choisis de $\mathrm{O}(3, k)$ on peut donc se ramener au cas où les points d'indétermination de $g$ et $g^{-1}$ sont tous deux $[1: 0: 0: 0]$. Ceci revient à dire qu'on s'est ramené au cas où la décomposition de $g$ commence et finit par un élément de $E_{G}$ (a priori à coefficients dans $\left.k(\delta)\right)$ :

$g=e_{n} \circ a_{n-1} \circ \cdots \circ a_{1} \circ e_{1}$ avec $a_{i} \in \mathrm{O}(3, k(\delta)) \backslash E_{G}, e_{j} \in E_{G} \backslash O(3, k(\delta))$. 
Une récurrence immédiate montre alors que $g$ s'écrit

$g:(x, y, z) \mapsto\left(\alpha^{2} \beta^{2 d_{1}+1} z^{\left(2 d_{1}+1\right) d_{2}}+\cdots, \alpha \beta^{d_{1}+1} z^{\left(d_{1}+1\right) d_{2}}+\cdots, \beta z^{d_{2}}+\cdots\right)$

avec $d_{1}, d_{2}>1$ (on a écrit seulement les composantes homogènes de plus haut degré). Comme $f$ est par hypothèse à coefficients dans $k$ on en déduit que $\beta$, et donc aussi $\alpha$, sont des éléments de $k$. En composant $g$ à gauche par l'automorphisme $(x, y, z) \mapsto\left(x-2 \alpha y z^{d_{1}}-\alpha^{2} z^{2 d_{1}+1}\right.$, $\left.y+\alpha z^{d_{1}+1}, z\right)$ qui est un élément de $E_{G}$ à coefficients dans $k$ on obtient un élément de $\operatorname{Aut}\left(V_{\lambda}\right)$ de degré strictement inférieur à celui de $g$. On conclut par récurrence sur le degré.

\subsection{Un théorème de Nagao.}

Le théorème suivant, dû à Nagao [10], décrit la structure du groupe $\mathrm{GL}_{2}$ à coefficients dans l'anneau de polynômes $k[X]$ :

Théorème 7 . Le groupe $\mathrm{GL}(2, k[X])$ est égal au produit amalgamé de $\mathrm{GL}(2, k)$ et du groupe triangulaire $\left\{\left(\begin{array}{cc}a & P(X) \\ 0 & d\end{array}\right) ; a, d \in k^{*}, P \in k[X]\right\} l e$ long de leur intersection.

Le groupe $\operatorname{PGL}(2, k[X])$ s'identifie de manière naturelle à certains automorphismes de $\mathbb{A}^{1} \times \mathbb{P}^{1}$ :

$$
\left(\begin{array}{ll}
a(X) & b(X) \\
c(X) & d(X)
\end{array}\right):(t, u) \mapsto\left(t, \frac{a(t) u+b(t)}{c(t) u+d(t)}\right) .
$$

De ce point de vue le théorème de Nagao (ou pour être très précis : la version du Théorème 7 pour $\operatorname{PGL}(2, k[X])$, qui lui est équivalente) est donné par le corollaire suivant du Théorème 5 :

Corollaire 8. Le groupe des automorphismes de $\mathbb{P}^{1} \times \mathbb{P}^{1} \backslash F$ qui fixent chaque droite $t=$ cte est égal au produit amalgamé du groupe

$$
\left\{(t, u) \mapsto\left(t, \frac{a u+b}{c u+d}\right) ;\left(\begin{array}{ll}
a & b \\
c & d
\end{array}\right) \in \operatorname{PGL}(2, k)\right\}
$$

et du groupe

$$
\left\{(t, u) \mapsto(t, \gamma u+P(t)) ; \gamma \in k^{*}, P \in k[X]\right\}
$$

le long de leur intersection. 
Cet énoncé s'obtient en quotientant les groupes mis en jeu dans le Théorème 5 par le groupe $\left\{(t, u) \mapsto(\alpha t+\beta, u) ; \alpha \in k^{*}, \beta \in k\right\}$.

\subsection{Preuve des Théorèmes 4 et 5 .}

Notre propos est de montrer comment notre preuve du théorème de Jung [6] s'adapte à ce nouveau contexte. Nous commençons par supposer que $k$ est un corps algébriquement clos. Dans toute la suite $C \subset \mathbb{P}^{1} \times \mathbb{P}^{1}$, appelée diviseur à l'infini, sera une courbe égale ou bien à la diagonale $D$ ou bien à la fibre $F$. On dit qu'une application birationnelle $g: X \rightarrow Y$ provient d'un automorphisme de $V=\mathbb{P}^{1} \times \mathbb{P}^{1} \backslash C$ si $X$ et $Y$ sont des compactifications de $V$ et que $g$ induit un automorphisme de $V$. On remarque tout d'abord qu'il existe des contraintes fortes sur les positions possibles des points d'indétermination de $g$ quand $Y=\mathbb{P}^{1} \times \mathbb{P}^{1}$ (c'est le fait que le diviseur à l'infini soit constitué d'une seule courbe irréductible qui est crucial).

Lemme 9. Soit $X$ une surface et $g$ une application birationnelle de $X$ dans $\mathbb{P}^{1} \times \mathbb{P}^{1}$ provenant d'un automorphisme de $V$. Nous supposons de plus que $g$ n'est pas un morphisme. Alors

1) $g$ admet un seul point d'indétermination propre, situé sur le diviseur à l'infini de $X$;

2) $g$ admet des points d'indétermination $p_{1}, \ldots, p_{r}(r \geq 1)$ tels que

(a) $p_{1}$ soit le point d'indétermination propre;

(b) pour tout $i=2, \ldots, r$, le point $p_{i}$ soit situé sur le diviseur produit en éclatant $p_{i-1}$;

3) chacune des courbes irréductibles contenues dans le diviseur à l'infini de $X$ est contractée sur un point par $g$;

4) avec les notations du théorème de Zariski appliqué à $g$ : la première courbe contractée par $\pi_{2}$ est la transformée stricte d'une courbe contenue dans le diviseur à l'infini de $X$;

5) en particulier, si $X=\mathbb{P}^{1} \times \mathbb{P}^{1}$, la première courbe contractée par $\pi_{2}$ est la transformée de la courbe $C$ «à la source».

Preuve: Il suffit de recopier la preuve du Lemme 9 dans [6]. 
Fixons maintenant $g$ un automorphisme de $V$, que l'on prolonge en une application birationnelle de $\mathbb{P}^{1} \times \mathbb{P}^{1}$ sur $\mathbb{P}^{1} \times \mathbb{P}^{1}$. D'après le Lemme 9.1 ) $g$ admet un unique point d'indétermination propre situé sur $C$. En composant $g$ par un élément de $A_{C}$ nous pouvons nous ramener au cas où ce point est $[1: 0],[1: 0]$. Cette hypothèse faite, nous allons maintenant montrer qu'il existe un diagramme

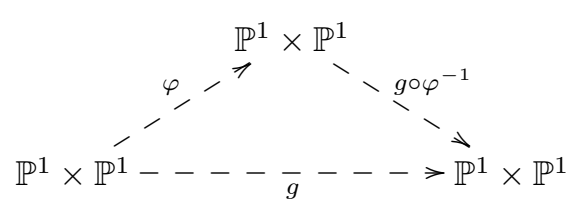

où $\varphi$ est le prolongement d'un élément de $E_{C}$, et tel que

$$
\# \operatorname{ind}\left(g \circ \varphi^{-1}\right)<\# \operatorname{ind}(g) \text {. }
$$

Par récurrence sur le nombre de points d'indétermination de $g$, on montre ainsi que cet automorphisme appartient au groupe engendré par $E_{C}$ et $A_{C}$. La question de la structure de produit amalgamé est traitée à part en fin d'article.

Cas $\boldsymbol{C}=\boldsymbol{F}$ : contexte du Théorème 5. Nous identifions $\mathbb{P}^{1} \times \mathbb{P}^{1}$ avec la surface de Hirzebruch $F_{0}$, et notons $f_{\infty}\left(F_{0}\right)=\left\{t_{1}=0\right\}$ et $s\left(F_{0}\right)=$ $\left\{u_{1}=0\right\}$. Remarquons que nous notons ici $s$ et non plus $s_{\infty}$ la section, en effet dans ce contexte le diviseur à l'infini est constitué de la seule courbe $f_{\infty}$. On se trouve alors dans les conditions du lemme suivant (cas ascendant, $n=0)$ :

Lemme 10. Soit $h: F_{n} \rightarrow \mathbb{P}^{1} \times \mathbb{P}^{1}$ provenant d'un automorphisme de $V$. Notons $p$ le point d'indétermination propre de $h, \varphi$ l'application birationnelle qui consiste à éclater $p$ puis à contracter la transformée de $f_{\infty}$, et $h^{\prime}=h \circ \varphi^{-1}$. Alors \#ind $\left(h^{\prime}\right)=\# \operatorname{ind}(h)-1$, et l'on distingue deux situations :

- cas ascendant : si $p=s \cap f_{\infty}$, alors on obtient une application $h^{\prime}: F_{n+1} \rightarrow \mathbb{P}^{1} \times \mathbb{P}^{1}$;

- cas descendant : si $p \neq s \cap f_{\infty}$, alors on obtient une application $h^{\prime}: F_{n-1} \rightarrow \mathbb{P}^{1} \times \mathbb{P}^{1}$. De plus le point d'indétermination $p^{\prime}$ de $h^{\prime}$ (si $h^{\prime} n^{\prime}$ est pas un morphisme) satisfait à nouveau $p^{\prime} \neq s\left(F_{n-1}\right) \cap$ $f_{\infty}\left(F_{n-1}\right)$.

Preuve: Considérons la décomposition de Zariski de $h$. Le Lemme 9.4) nous assure que la première courbe contractée par $\pi_{2}$ est la transformée de $f_{\infty}$. Le point $p$, unique point d'indétermination propre de $h$, est donc 
contenu dans $f_{\infty}$. Après avoir éclaté $p, f_{\infty}$ est déjà devenue d'autointersection -1 ; on en déduit que tous les autres éclatements de la suite $\pi_{1}$ concernent des points hors de $f_{\infty}$. Il revient donc au même de réaliser ces éclatements puis de contracter $f_{\infty}$, ou de contracter d'abord $f_{\infty}$ puis de réaliser les éclatements. Ceci revient à dire que l'on a un diagramme commutatif :

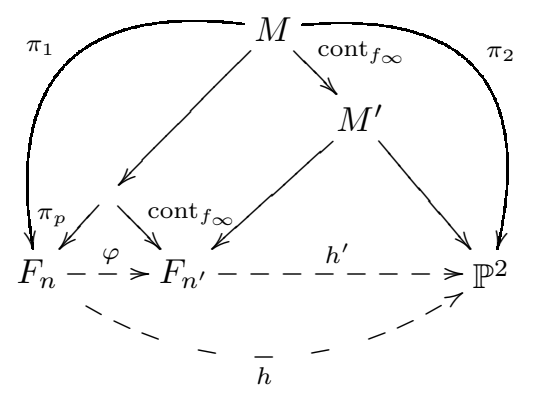

L'égalité \#ind $\left(h^{\prime}\right)=\# \operatorname{ind}(h)-1$ en découle; et la distinction entre les cas ascendant et descendant n'est plus alors qu'une remarque immédiate.

Après avoir appliqué une première fois le lemme (cas ascendant, $n=0$ ), on se retrouve à nouveau dans les conditions du lemme avec $n=1$. On applique ainsi le lemme autant de fois que l'on se trouve dans le cas ascendant (disons $r$ fois, avec $r \geq 1$ ). On obtient le diagramme

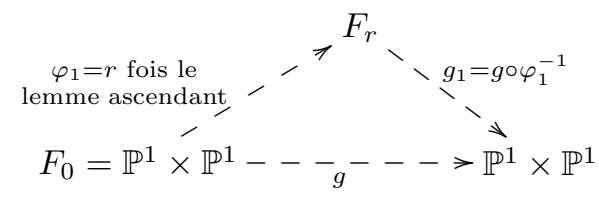

où $g_{1}$ satisfait les conditions du lemme (avec $n=r$, cas descendant), et $\# \operatorname{ind}\left(g_{1}\right)=\# \operatorname{ind}(g)-r$. On peut alors appliquer successivement $r$ fois le lemme, cas descendant, jusqu'à obtenir le diagramme :

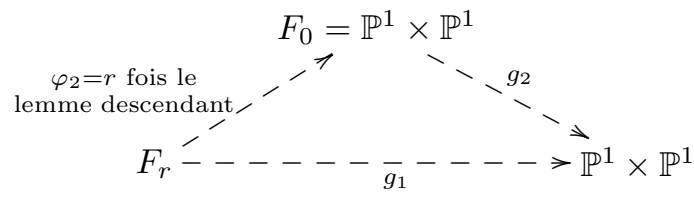


En conclusion, on a obtenu un diagramme

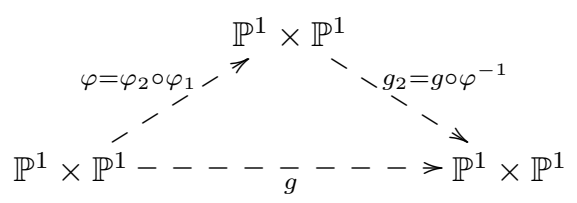

avec \#ind $\left(g \circ \varphi^{-1}\right)=\# \operatorname{ind}(g)-2 r$. Reste à vérifier que $\varphi \in E_{F}$. Pour cela, il suffit de remarquer que $\varphi$ induit un automorphisme de $\mathbb{A}^{2}=$ $\mathbb{P}^{1} \times \mathbb{P}^{1} \backslash\left(s \cup f_{\infty}\right)$ qui préserve la fibration $t=$ cte, et ceci caractérise les automorphismes élémentaires.

Cas $\boldsymbol{F}=\boldsymbol{D}$ : contexte du Théorème 4. Reprenons le diagramme de Zariski associé à $g$. Par le Lemme 9.5), nous savons que la première courbe contractée par $\pi_{2}$ sera la transformée de $D$. Or $D$ est d'autointersection +2 dans $\mathbb{P}^{1} \times \mathbb{P}^{1}$ et doit donc subir trois éclatements afin de devenir d'auto-intersection -1. Ceci implique qu'il y a une unique possibilité pour les 3 premiers éclatements de la suite $\pi_{1}$, que nous allons expliciter. Après avoir éclaté le point d'indétermination propre $[1: 0],[1: 0]$, le point d'intersection du diviseur exceptionnel et de la transformée de $D$ est obligatoirement le deuxième point d'indétermination de $g$. Eclatons donc ce point : on obtient une surface $\Sigma_{1}$ et $g$ se factorise à travers ces deux éclatements en une application $g_{1}: \Sigma_{1} \rightarrow \mathbb{P}^{1} \times \mathbb{P}^{1}$ avec $\# \operatorname{ind}\left(g_{1}\right)=\# \operatorname{ind}(g)-2$. Remarquons que $\Sigma_{1}$ peut être vue comme $F_{1}$ éclaté deux fois (contracter les transformées des deux fibres de $F_{0}$ qui passaient par $[1: 0],[1: 0])$ : la fibration sur $F_{1}$ correspond à la fibration sur $\Sigma_{1}$ donnée par les droites $t+u=$ cte. De manière générale nous noterons $\Sigma_{n}$ une surface compacte contenant un ouvert isomorphe à $V=\mathbb{P}^{1} \times \mathbb{P}^{1} \backslash D$, et telle que $\Sigma_{n} \backslash V$ soit constitué de trois courbes rationnelles d'auto-intersection $0,-n$ et -2 disposées comme suit :

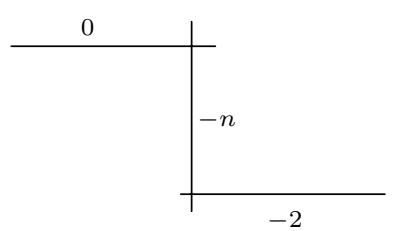

On peut voir $\Sigma_{n}$ comme la surface obtenue en éclatant une surface de Hirzebruch $F_{n}$ en deux points situés sur une même fibre (et non inclus dans $\left.f_{\infty} \cup s_{\infty}\right)$. Nous continuons à noter $s_{\infty}$ et $f_{\infty}$ les courbes correspondantes : 
$F_{n}$

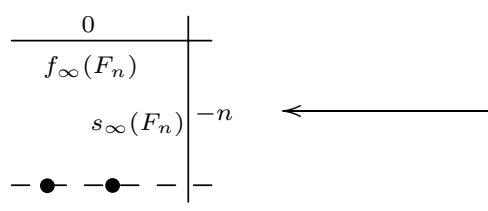

$\Sigma_{n}$

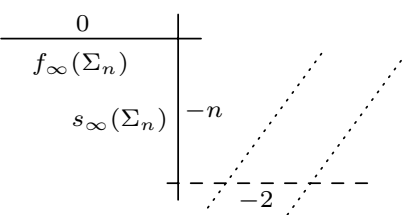

Ces notations étant posées, remarquons que le point d'indétermination propre de $g_{1}$, qui correspond au troisième point d'indétermination de $g$, est forcément le point d'intersection de $s_{\infty}\left(\Sigma_{1}\right)$ et de $f_{\infty}\left(\Sigma_{1}\right)$. Nous sommes ainsi dans les conditions du lemme suivant (avec $n=1$, cas ascendant) :

Lemme 11. Soit $h: \Sigma_{n} \rightarrow \mathbb{P}^{1} \times \mathbb{P}^{1}$ le prolongement d'un automorphisme de $V$, on note $p$ le point d'indétermination propre de $h$.

- cas ascendant : supposons que $n \geq 1$ et $p=s_{\infty} \cap f_{\infty}$. Alors en éclatant $p$ et en contractant la transformée de $f_{\infty}$ on obtient une application $h^{\prime}: \Sigma_{n+1} \rightarrow \mathbb{P}^{1} \times \mathbb{P}^{1}$;

- cas descendant : supposons que $n \geq 2$ et que $p \neq s_{\infty} \cap f_{\infty}$. Alors $p \in f_{\infty}$, et en éclatant $p$ et en contractant la transformée de $f_{\infty}$ on obtient une application $h^{\prime}: \Sigma_{n-1} \rightarrow \mathbb{P}^{1} \times \mathbb{P}^{1}$.

De plus dans les deux cas \#ind $\left(h^{\prime}\right)=\# \operatorname{ind}(h)-1$.

Preuve: Appliquons le théorème de Zariski à $h$. La première courbe contractée par $\pi_{2}$ est la transformée de l'une des trois courbes à l'infini par le Lemme 9.4) : c'est donc la transformée de $f_{\infty}$, car les deux autres sont d'auto-intersection strictement inférieure à -1 dans $M$. On conclut en reprenant l'argument déjà détaillé dans la preuve du Lemme 10.

On applique le lemme, cas ascendant, autant de fois que possible (disons $r$ fois) jusqu'à se retrouver dans les conditions du lemme, cas descendant. On peut alors appliquer $r$ fois le lemme, cas descendant, jusqu'à obtenir une application $g_{2}: \Sigma_{1} \mapsto \mathbb{P}^{1} \times \mathbb{P}^{1}$, avec \#ind $\left(g_{2}\right)=\# \operatorname{ind}\left(g_{1}\right)-2 r$. On contracte alors deux fois pour arriver à $\bar{g}: \mathbb{P}^{1} \times \mathbb{P}^{1} \mapsto \mathbb{P}^{1} \times \mathbb{P}^{1}$. Il est immédiat que \#ind $(\bar{g}) \leq \# \operatorname{ind}\left(g_{2}\right)+2$; en fait on pourrait montrer que $\# \operatorname{ind}(\bar{g})=\# \operatorname{ind}\left(g_{2}\right)$. Toujours est-il que même avec cette estimation grossière on a encore :

$$
\# \operatorname{ind}(\bar{g}) \leq \# \operatorname{ind}(g)-2 r \text {. }
$$

Reste à vérifier que l'application $\bar{g}^{-1} \circ g$ est un élément de $E_{D}$ : pour cela il suffit de constater que cette application préserve le pinceau des 
droites $t+u=$ cte, celui-ci correspondant à la fibration de chacune des surfaces $\Sigma_{n}$ mises en jeu au cours de la preuve.

Fin des preuves. Nous discutons maintenant les deux points manquants pour compléter la preuve des Théorèmes 4 et 5 : d'une part la structure de produit amalgamé, d'autre part la preuve dans le cas d'un corps quelconque.

Structure de produit amalgamé (comparer avec $[6,4.2])$. Posons $p=[1: 0],[1: 0]$. Alors pour $C=D$ ou $C=F$ on a :

1) tout élément de $E_{C} \backslash A_{C}$, vu comme une application $\mathbb{P}^{1} \times \mathbb{P}^{1} \rightarrow$ $\mathbb{P}^{1} \times \mathbb{P}^{1}$, admet $p$ comme unique point d'indétermination propre, et contracte $C$ sur $p$;

2) tout élément de $A_{C} \backslash E_{C}$ ne fixe pas le point $p$.

On constate ainsi que toute composition (non réduite à un élément de $A_{C}$ ) d'éléments de $E_{C} \backslash A_{C}$ et $A_{C} \backslash E_{C}$ contracte la courbe $C$ sur un point, et n'est donc pas égale à l'identité.

Cas d'un corps quelconque. Soit $k$ un corps, et $\bar{k}$ sa clôture algébrique. Vérifions que le Lemme 9 est encore valable sur $k$, et en particulier le point clé que constitue l'affirmation 9.1). Considérons donc $g$ une application birationnelle de $X_{k}$ dans $\mathbb{P}_{k}^{1} \times \mathbb{P}_{k}^{1}$ provenant d'un automorphisme algébrique de $\mathbb{P}_{k}^{1} \times \mathbb{P}_{k}^{1} \backslash C$. Les points d'indéterminations de $g$, qui a priori sont des points sur $\bar{k}$, sont invariants sous l'action du groupe de Galois $\operatorname{Gal}(\bar{k} / k)$. Mais nous savons (c'est le Lemme 9 appliqué sur $\bar{k}$ ) que $g$ admet un unique point d'indétermination propre $p_{0}$. Ce point est donc invariant sous l'action de $\operatorname{Gal}(\bar{k} / k)$, autrement dit $p_{0}$ est un point sur $k$. On en déduit immédiatement les autres affirmations du lemme. En conclusion les preuves restent valables car tous les éclatements réalisés au cours de la démonstration concernent des points sur $k$.

\section{Applications polynomiales propres de $\mathbb{C}^{2}$}

Dans ce paragraphe nous nous proposons de discuter une généralisation possible du résultat de Jung-Van der Kulk. Un automorphisme polynomial de $\mathbb{C}^{2}$ peut être caractérisé comme un morphisme propre de $\mathbb{C}^{2}$ dans $\mathbb{C}^{2}$ de degré topologique 1 . En conservant l'hypothèse de propreté mais en considérant des applications de degré topologique arbitraire on obtient le semi-groupe des applications polynomiales propres de $\mathbb{C}^{2}$ dans $\mathbb{C}^{2}$. On sait qu'une application polynomiale propre ne peut candidater à être un contre-exemple à la conjecture du jacobien $[\mathbf{1}$, 
Th. 2.1]. Ceci explique peut-être le modeste intérêt porté à ce type d'applications dans la littérature. Il me semble pourtant qu'il s'agit d'une classe d'applications pouvant présenter des comportements dynamiques nouveaux par rapport au cas des automorphismes, tout en se prêtant encore à une étude un tant soit peu exhaustive (dans cet esprit signalons la prépublication [2] qui traite de la dynamique d'applications « à allure polynomiale »; cependant comme le remarquent les auteurs p. 18 en général un endomorphisme polynomial de $\mathbb{C}^{k}$ avec $k \geq 2$ n'est pas à allure polynomiale même s'il est propre »).

Nous considérons donc maintenant des applications polynomiales de $\mathbb{C}^{2}$ dans $\mathbb{C}^{2}$ de degré topologique quelconque qui soient propres (la préimage d'un compact est un compact). Bien sûr les éléments de Aut $\left[\mathbb{C}^{2}\right]$ fournissent des exemples. Une autre classe d'exemples très simples est donnée par les applications de la forme

$$
(x, y) \mapsto(P(x), Q(y)) .
$$

Un contre-exemple évident est l'application $(x, y) \mapsto(x y, y)$, en effet la préimage de tous voisinage de $(0,0)$ contient la droite $y=0$. Un contreexemple plus subtil (qui m'a été indiqué par J.-P. Furter) est fourni par l'application $(x, y) \mapsto\left(x+x^{2} y, y\right)$. En effet la préimage de tout point est constituée d'un ou deux points mais l'application n'est pas propre : les points $(-1 / \varepsilon, \varepsilon)$ et $(0, \varepsilon)$ sont les deux préimages de $(0, \varepsilon)$, en conséquence la préimage d'un voisinage compact de $(0,0)$ n'est jamais compacte. Nous ne connaissons pas d'autres exemples d'applications polynomiales propres que ceux cités ci-dessus (sauf à les composer) : on songe immédiatement à conjecturer que ce sont les seuls... Disons simplement que nous posons la

Question. Est-ce que toutes les applications polynomiales propres de $\mathbb{C}^{2}$ dans $\mathbb{C}^{2}$ sont des composées d'automorphismes polynomiaux et d'applications de la forme $(x, y) \mapsto(x, P(y))$ ?

Nous n'avons pu répondre à cette question que dans le cas du degré topologique 2 ; c'est ce que nous exposons maintenant. Notons donc $g: \mathbb{C}^{2} \mapsto \mathbb{C}^{2}$ une application polynomiale propre de degré topologique 2 . Associons à $g$ l'involution $\sigma$ qui échange les deux préimages d'un point (éventuellement ces deux préimages sont confondues et définissent alors un point fixe pour $\sigma$ ).

Lemme 12. L'involution $\sigma$ est un élément de Aut $\left[\mathbb{C}^{2}\right]$. En particulier $\sigma$ est conjuguée dans Aut $\left[\mathbb{C}^{2}\right]$ ou bien à $(-x,-y)$ ou bien à $(x,-y)$.

Preuve: Considérons le prolongement $g: \mathbb{P}^{2} \rightarrow \mathbb{P}^{2}$. En éclatant suffisamment de fois au but on peut supposer que la droite à l'infini à la 
source n'est pas contractée sur un point, c'est à dire que l'on a un diagramme

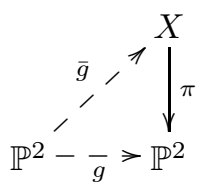

où $\bar{g}$ ne contracte aucune courbe (ni la droite à l'infini par construction, ni une autre courbe par hypothèse de propreté). Associons comme ci-dessus une involution à $\bar{g}$, c'est une application holomorphe qui prolonge $\sigma$ et est bien définie en dehors du nombre fini de points d'indétermination de $\bar{g}$ : par Hartogs $\sigma$ est une application birationnelle de $\mathbb{P}^{2}$ dans $\mathbb{P}^{2}$. Comme de plus $\sigma$ envoie $\mathbb{C}^{2}$ sur $\mathbb{C}^{2}$ (c'est à nouveau l'hypothèse de propreté), on a bien $\sigma \in \operatorname{Aut}\left[\mathbb{C}^{2}\right]$.

On sait qu'un élément d'ordre fini de $\operatorname{Aut}\left[\mathbb{C}^{2}\right]$, et donc en particulier une involution, est conjugué à un automorphisme élémentaire. De plus (voir [3]), tout automorphisme élémentaire est conjugué :

1) ou bien à un élément du groupe affine;

2) ou bien à un élément de la forme $\left(\beta^{d} x+\beta^{d} y^{d} q\left(y^{r}\right), \beta y\right)$ avec $d \geq 1$, $q$ non constant, $\beta$ racine rième de l'unité.

On remarque que dans le deuxième cas l'automorphisme est toujours d'ordre infini. Finalement $\sigma$ est conjugué à un automorphisme affine. Il est facile de vérifier que $\sigma$ admet un point fixe; en conjuguant par une translation puis en diagonalisant on a le résultat annoncé.

Nous sommes maintenant en mesure de montrer la

Proposition 13. Soit $g: \mathbb{C}^{2} \mapsto \mathbb{C}^{2}$ une application polynomiale propre de degré topologique 2 . Alors il existe $f_{1}, f_{2} \in \mathrm{Aut}\left[\mathbb{C}^{2}\right]$ tels que g s'écrive

$$
g=f_{2} \circ\left(x, y^{2}\right) \circ f_{1} \text {. }
$$

Preuve: Considérons l'involution $\sigma \in$ Aut $\left[\mathbb{C}^{2}\right]$ associée à $g$, et notons $f_{1}$ l'automorphisme fourni par le Lemme 12 tel que $\sigma^{\prime}=f_{1}^{-1} \sigma f_{1}=(x,-y)$ ou $(-x,-y)$. Posons $g^{\prime}=g \circ f_{1}$, on a $g^{\prime} \circ \sigma^{\prime}=g^{\prime}$. Supposons $\sigma^{\prime}=(x,-y)$. Alors cela signifie que $g^{\prime}$ ne dépend que de $x$ et $y^{2}$, autrement dit il existe une application polynomiale $f_{2}: \mathbb{C}^{2} \mapsto \mathbb{C}^{2}$ telle que $g^{\prime}=f_{2} \circ\left(x, y^{2}\right)$. De plus $f_{2}$ est propre car $g^{\prime}$ est propre, et $f_{2}$ est de degré topologique 1 car $g^{\prime}$ et $\left(x, y^{2}\right)$ sont de degré topologique 2 . Finalement $f_{2} \in \operatorname{Aut}\left[\mathbb{C}^{2}\right]$, et on a bien $g=f_{2} \circ\left(x, y^{2}\right) \circ f_{1}$. Il est facile de voir que le cas $\sigma^{\prime}=(-x,-y)$ est impossible, en effet on aurait $g^{\prime}=f_{2} \circ\left(x^{2}, y^{2}\right)$ mais $g^{\prime}$ est de degré topologique 2 alors que $\left(x^{2}, y^{2}\right)$ est de degré 4 . 


\section{Références}

[1] H. Bass, E. H. Connell et D. Wright, The Jacobian conjecture : reduction of degree and formal expansion of the inverse, Bull. Amer. Math. Soc. (N.S.) 7(2) (1982), 287-330.

[2] T.-C. Dinh ET N. Sibony, Dynamique des applications d'allure polynomiale, J. Math. Pures Appl. (9) 82(4) (2003), 367-423.

[3] S. Friedland ET J. Milnor, Dynamical properties of plane polynomial automorphisms, Ergodic Theory Dynam. Systems 9(1) (1989), 67-99.

[4] M. H. Gizatullin et V. I. Danilov, Automorphisms of affine surfaces. II, (Russian), Izv. Akad. Nauk SSSR Ser. Mat. 41(1) (1977), 54-103, 231.

[5] R. Hartshorne, "Algebraic geometry", Graduate Texts in Mathematics 52, Springer-Verlag, New York-Heidelberg, 1977.

[6] S. LAmY, Une preuve géométrique du théorème de Jung, Enseign. Math. (2) 48(3-4) (2002), 291-315.

[7] S. LAmy, Automorphismes polynomiaux préservant une action de groupe, Bol. Soc. Mat. Mexicana (3) 9(1) (2003), 1-19.

[8] L. MAKAR-Limanov, On groups of automorphisms of a class of surfaces, Israel J. Math. 69(2) (1990), 250-256.

[9] A. W. Mason, Serre's generalization of Nagao's theorem : an elementary approach, Trans. Amer. Math. Soc. 353(2) (2001), 749-767 (electronic).

[10] H. Nagao, On GL(2,K[x]), J. Inst. Polytech. Osaka City Univ. Ser. A 10 (1959), 117-121.

[11] J.-P. SERre, Arbres, amalgames, SL 2 , Astérisque 46 (1977), Société Mathématique de France, Paris, 189 pp.

Université Lyon 1

Institut Girard Desargues

21 Avenue Claude Bernard

69622 Villeurbanne Cedex

France

E-mail address: lamy@igd.univ-lyon1.fr

Primera versió rebuda el 22 d'octubre de 2003, darrera versió rebuda el 9 de novembre de 2004. 